\title{
Lymphoepithelioma-like carcinoma of the lung in a patient with silicosis
}

\author{
J.C. Ho*, W.K. Lam*, G.C. Ooi\#, M.P. Wong", J.C. Lam*, M.S. Ip*, K.W.T. Tsang*
}

Lymphoepithelioma-like carcinoma of the lung in a patient with silicosis. J.C. Ho, W.K. Lam, G.C. Ooi, M.P. Wong, J.C. Lam, M.S. Ip, K.W.T. Tsang. (CERS Journals Ltd 2003.

ABSTRACT: Silicosis is an important form of pneumoconiosis, which is caused by significant exposure to crystalline silica. The carcinogenicity of silica, despite traditional beliefs, in relation to lung cancer has been controversial. Lymphoepitheliomalike carcinoma of the lung, an Epstein-Barr virus-associated undifferentiated carcinoma, is a rare entity of pulmonary malignancy, which tends to affect young nonsmoking Asians.

The first case of silicosis, initially complicated by pulmonary tuberculosis, which later developed into advanced lymphoepithelioma-like carcinoma of the lung is reported. A combination of 5-fluorouracil, cisplatin and calcium folinate resulted in partial tumour response.

Eur Respir J 2003; 22: 383-386.
University Depts of *Medicine, "Diagnostic Radiology and "Pathology, The University of Hong Kong, Queen Mary Hospital, Hong Kong SAR, China.

Correspondence: K.W.T. Tsang, Division of Respiratory and Critical Care Medicine, University Dept of Medicine, The University of Hong Kong, Queen Mary Hospital, Pokfulam, Hong Kong SAR, China.

Fax: 85229049443

E-mail: kwttsang@hkucc.hku.hk

Keywords: Chemotherapy lymphoepithelioma

silicosis

Received: December 112002

Accepted: February 172003
Inhalation of dusts is an important cause of interstitial lung disease in many countries. Silicosis, pneumoconiosis of coal workers, and asbestosis are the three most commonly recognised forms of pneumoconiotic pulmonary fibrosis [1]. Although the pathogenetic role of crystalline silica in silicosis has been well described [2-4], the association between silica exposure and lung cancer remains controversial $[5,6]$.

BEGIN et al. [7] first reported lymphoepithelioma-like carcinoma (LELC) of the lung in 1987. LELC of the lung tends to affect young nonsmoking patients and the mean age of affected patients was reported to be 10 yrs younger than that in other histological types of nonsmall cell lung carcinoma in a Taiwanese series [8]. The mean age was 48 yrs in a Hong Kong series [9]. Probably due to the racial distribution of LELC, most of the major series on LELC of the lung have been reported from Southern China $[8,10,11]$. Pathological, epidemiological and histopathological studies have unequivocally shown an association between LELC of the lung and Epstein-Barr virus (EBV) infection [8, 9, 12-18]. However, the treatment of LELC is controversial, and little is known regarding the precise role of chemotherapy and radiotherapy in this rare condition $[10,12-14,19,20]$.

The first case of LELC of the lung occurring in a patient with silicosis is reported. This rare association between silicosis and a nonsmoking-related lung cancer lends support to a carcinogenic role of silica. The efficacy of combination chemotherapy (5-fluorouracil, cisplatin and calcium folinate) has also been demonstrated in this case [20].

\section{Case report}

A 71-yr-old exsmoker (cumulative cigarette consumption 25 pack-yrs), who was an iron miner for $>10 \mathrm{yrs}$, was diagnosed as having silicosis, based on radiographical features and a history of dust exposure, in 1987. He presented with haemoptysis, cough and weight loss in 1999. Chest radiography revealed diffuse nodular shadows in both lungs consistent with silicosis and a right middle lobe mass (fig. 1). Radiologically, the mass could still have represented atypical progressive massive fibrosis, although neoplastic or infective lesions had to be excluded. There were no other symptoms or

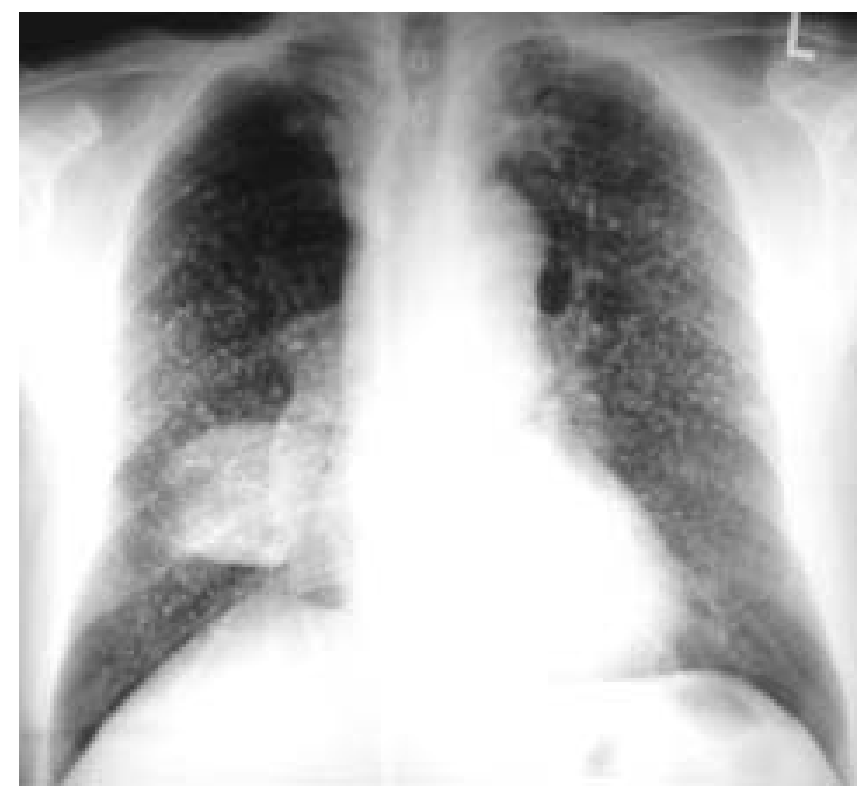

Fig. 1.-Chest radiograph showing diffuse nodular shadows over both lungs and a right middle lobe mass. 


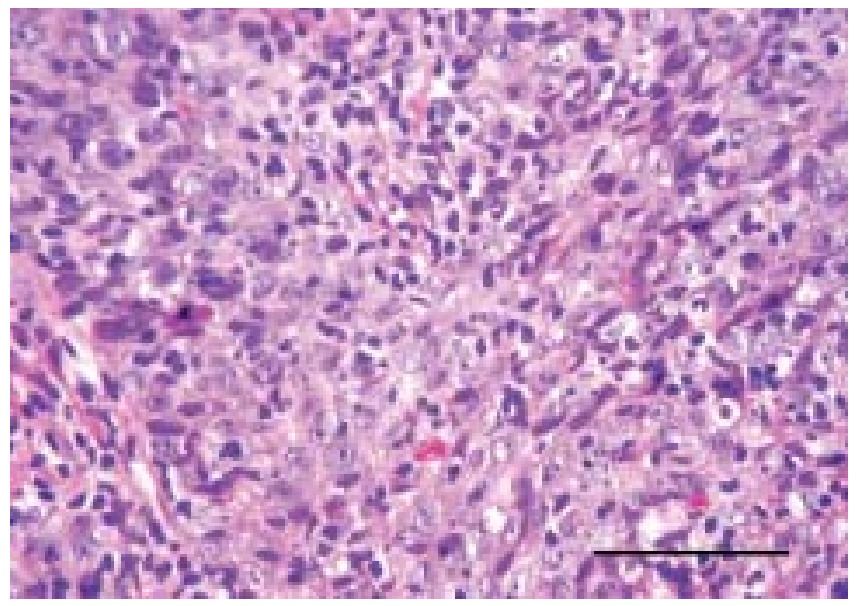

Fig. 2.-Bronchial biopsy specimen showing fragments of cohesive tumour cells arranged in syncytial sheets and intermixed with lymphoplasmacytic inflammatory cells. The tumour cells show oval nuclei, prominent nucleoli and indistinct cytoplasmic borders. Haematoxylin and eosin stain; internal scale bar $=250 \mu \mathrm{m}$

signs on detailed history evaluation and physical examination. The patient underwent fibreoptic bronchoscopy, the results of which were macroscopically normal. Bronchoalveolar lavage fluid (BALF) gave negative acid-fast bacilli stain results. Percutaneous transthoracic needle biopsy (PTNB) of the mass lesion, performed under computed tomographic guidance, showed small noncaseating epithelioid granulomata, with

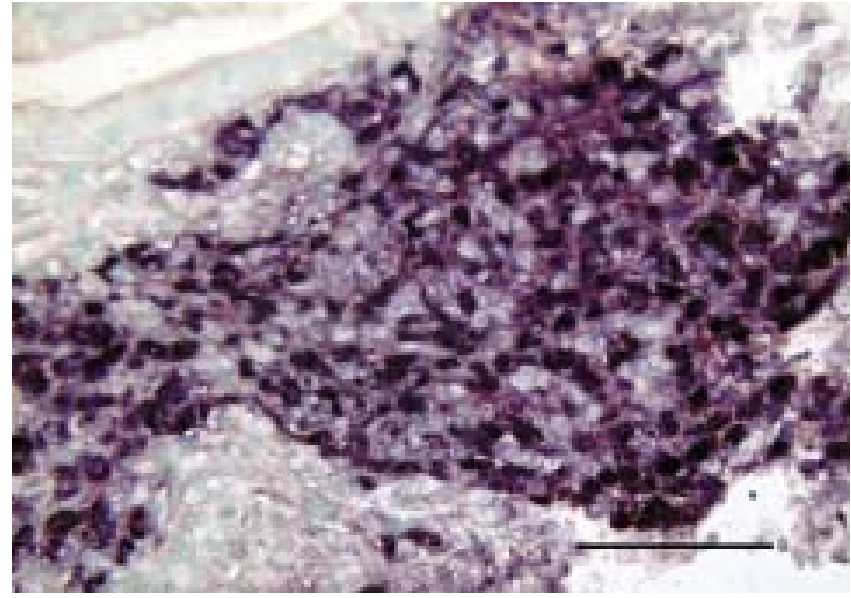

Fig. 3. - In situ hybridisation study showing strong positivity for Epstein-Barr virus- encoded small ribonucleic acids in tumour nuclei, in contrast to negative signals in the lymphocytes. Internal scale bar $=250 \mu \mathrm{m}$

positive Ziehl-Neelsen staining for acid-fast bacilli. Standard antituberculous treatment ( 2 months of isoniazid, rifampicin, pyrazinamide and ethambutol, followed by another 7 months of isoniazid and rifampicin) was commenced. Subsequent microbiological culture of BALF confirmed the presence of Mycobacteria tuberculosis with favourable sensitivity tests to the above first-line antituberculous drugs.
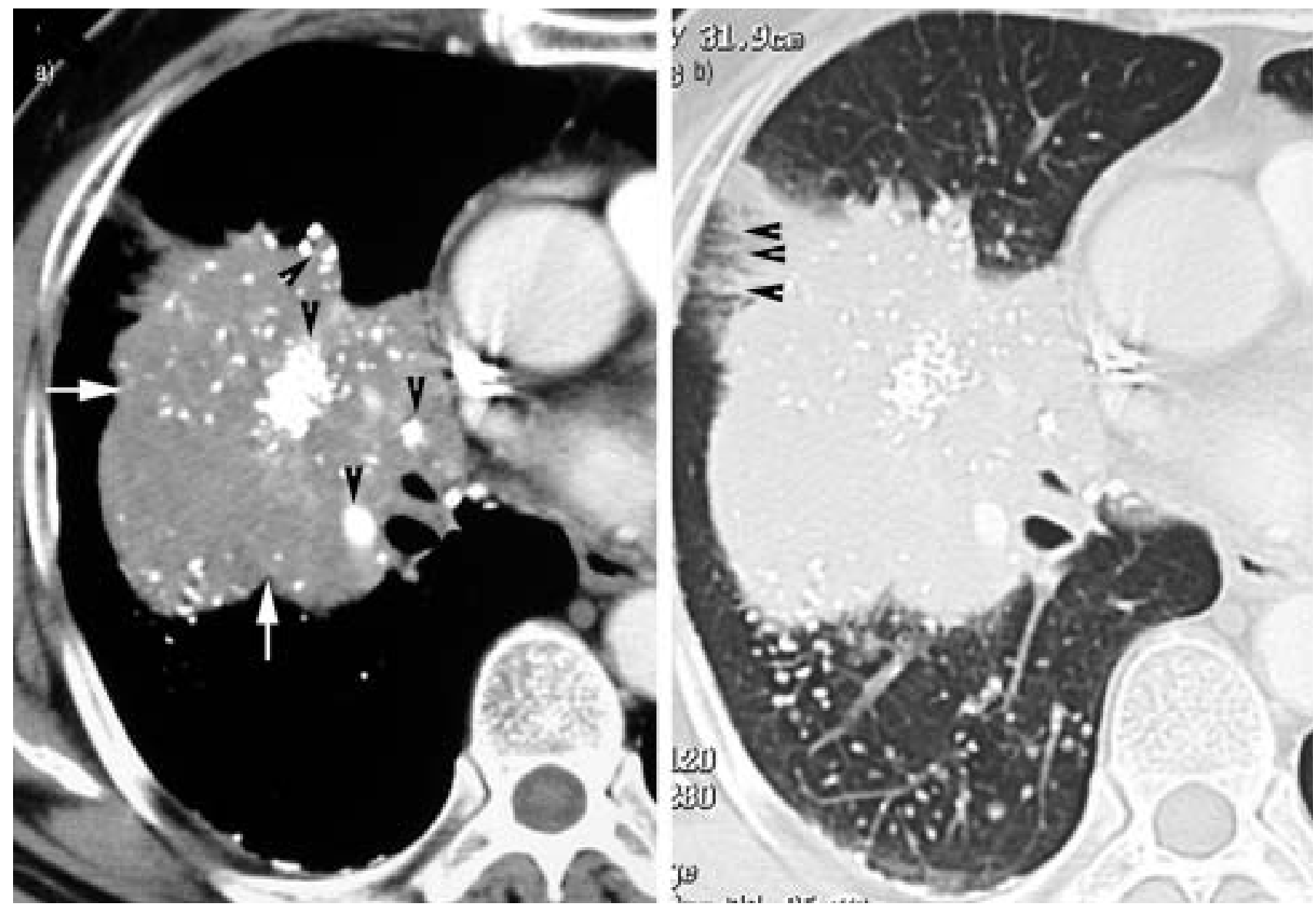

Fig. 4. - Contrast-enhanced computed tomography scan of the thorax showing a) an $8.2 \times 8.8 \times 6.5$-cm mass in the right middle lobe (arrows) with amorphous and eccentric calcification (arrowheads). b) The borders are relatively well-defined apart from spiculation at the anterior border (arrowheads). Multiple noncalcified silicotic lung nodules are also present. 
However, there was no improvement in radiographical appearance after completion of the antituberculous treatment, despite the lack of respiratory or other systemic symptoms. One year after completion of antituberculous treatment, the right middle lobe mass progressively enlarged on follow-up chest radiographs. Fibreoptic bronchoscopy showed a tumour at the right middle lobe bronchus. Bronchial biopsy revealed fragments of cohesive tumour cells arranged in syncytial sheets, and intermixed with lymphoplasmacytic inflammatory cells. The tumour cells had plump-spindled hyperchromatic nuclei, distinct nucleoli and indistinct cytoplasmic borders (fig. 2). In situ hybridisation was strongly positive for EBV-encoded small ribonucleic acids (RNAs), compatible with LELC (fig. 3). PTNB at the right middle lobe mass revealed a silicotic nodule and nests of infiltrating tumour. Computed tomography $(\mathrm{CT})$ of the thorax showed an $8.2 \times 8.8 \times 6.5-\mathrm{cm}$ mass in the right middle lobe with eccentric and amorphous calcification, against a background of multiple noncalcified silicotic lung nodules and calcified mediastinal lymph nodes (fig. 4). It was relatively well defined apart from its anterior borders, which were spiculated. Encasement of the right descending pulmonary artery and right middle lobe bronchus was present. An 18-fluorodeoxyglucose positron emission tomography scan showed a hypermetabolic tumour in the right middle lobe, with diffuse increased activity in multiple areas of the mediastinum, suggesting extensive malignant mediastinal lymphadenopathy (radiologically staged IIIA; tumour 3 , node 2 , metastasis 0 ). As a routine investigation for LELC of the lung and to exclude a nasopharyngeal origin of the tumour, nasopharyngeal examination and magnetic resonance imaging of the nasopharynx were performed, which revealed no abnormalities. Anti-EBV immunoglobulin A serology (enzyme-linked immunosorbent assay) was negative.

Systemic chemotherapy, consisting of four 4-weekly cycles of 5-fluorouracil $\left(1,000 \mathrm{mg} \cdot \mathrm{m}^{-2}\right.$ body surface area $(B S A) \cdot$ day $^{-1}$ on days $1-4)$, calcium folinate $\left(200 \mathrm{mg} \cdot \mathrm{m}^{-2} \mathrm{BSA} \cdot \mathrm{day}^{-1}\right.$ on days $1-4)$ and cisplatin $\left(100 \mathrm{mg} \cdot \mathrm{m}^{-2} \mathrm{BSA} \cdot \mathrm{day}^{-1}\right.$ on day 1$)$, was commenced. After completion of four cycles of chemotherapy, the tumour had significantly reduced in size, i.e. $>50 \%$ compared with baseline CT scan (fig. 5). The chemotherapy was well tolerated with only mild anaemia, thrombocytopenia, nausea, vomiting and stomatitis.

\section{Discussion}

The present patient illustrates the commonly encountered difficulty in the management of silicotic patients who develop new lung shadows, the diagnosis of which may be confused with complications associated with the silicotic process such as tuberculosis or progressive massive fibrosis. Silicosis is an important form of pneumoconiotic pulmonary fibrosis related to exposure to silica dusts. Pulmonary tuberculosis represents a common complication seen in up to $50 \%$ of patients with silicosis in some clinical series [1]. However, the relationship between silicosis and lung cancer, despite the adoption of crystalline silica as a group 1 human carcinogen by the International Agency for Research on Cancer, is still controversial $[5,6]$. Tumour suppressor $\mathrm{p} 53$ gene messenger RNA is suppressed by intratracheal injection with crystalline silica in mice [21]. The mutation frequencies of the p53 gene were high among lung cancers in silicotics, but the mutation distributions in exons differed from those without silicosis [22]. However, silica has not been shown to be directly genotoxic and has only been proven to be a pulmonary carcinogen in rats [6]. Epidemiological studies on the carcinogenicity of silica have shown inconsistent and sometimes even contradictory results [23]. Apart from factors related to

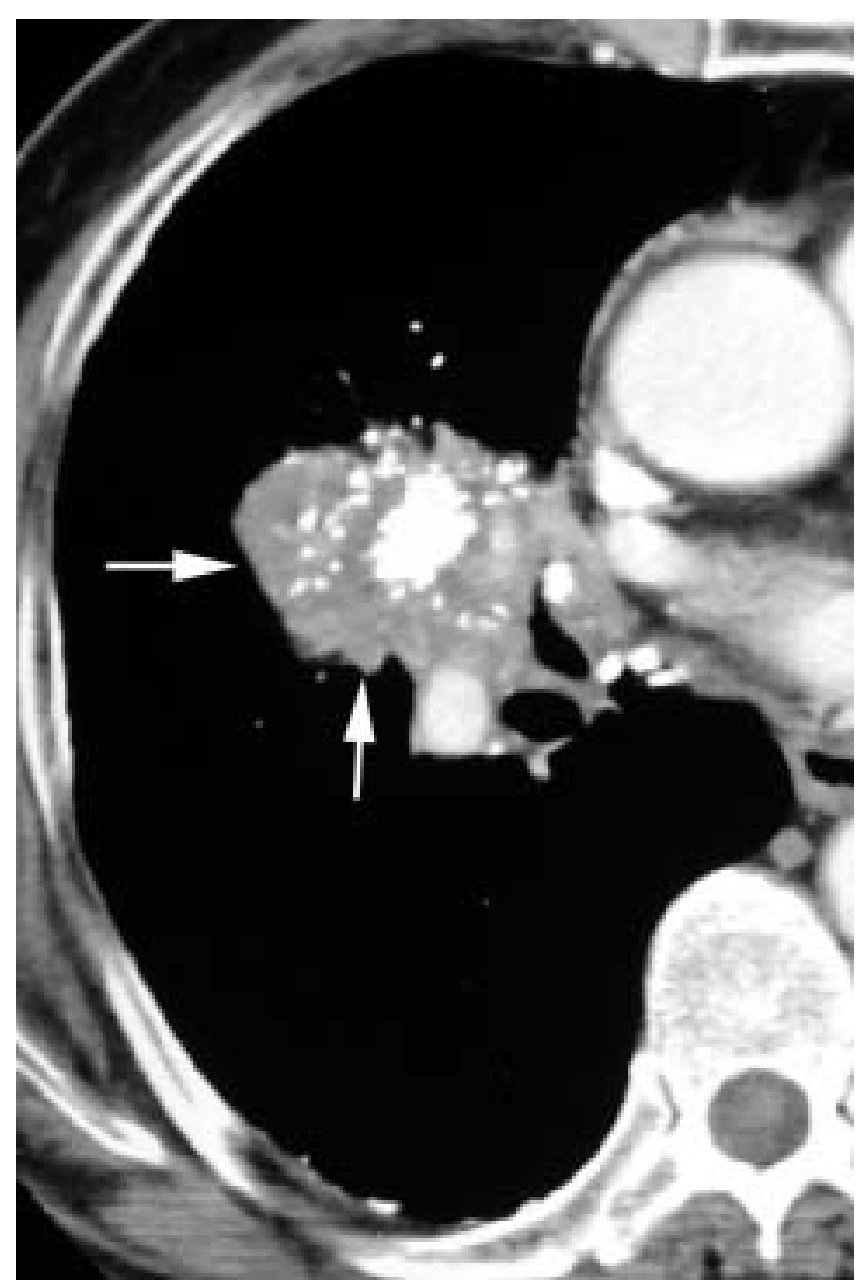

Fig. 5.-Contrast-enhanced computed tomography scan of the thorax showing a $>50 \%$ reduction in tumour size (arrows) after completion of four courses of systemic chemotherapy.

study design and concomitant exposure to other potential carcinogens such as cadmium, polycyclic aromatic hydrocarbons [24] and radon daughters [25], smoking has been a major confounding and uneliminated factor in these studies [25].

LELC is pathologically a distinct entity classified as a type of nonsmall cell lung cancer [8,9]. Primary LELC of the lung is histologically indistinguishable from the prototypical LELC occurring in the nasopharynx [8], and it is therefore imperative that a nasopharyngeal origin is actively excluded in all cases. Major differential diagnoses for LELC are nonHodgkin's lymphoma and metastatic nasopharyngeal carcinoma, both of which are common among the Chinese [12]. Immunohistochemical staining helps to differentiate lymphoma from LELC [8]. Endoscopic examination and random biopsy of the nasopharynx together with CT or preferably magnetic resonance imaging are often necessary to exclude primary nasopharyngeal carcinoma.

Experience with systemic chemotherapy in the treatment of LELC of the lung is limited. Postoperative chemotherapy with four cycles of carboplatin and etoposide (VP-16) had been used for stage II LELC of the lung in one report [13]. There was another report of induction chemotherapy consisting of 5-fluorouracil, calcium folinate and cisplatin in a child with LELC of the lung resulting in significant tumour reduction [19]. In a series of advanced LELC of the lung treated with palliative chemotherapy comprising 5-fluorouracil and 
cisplatin, 71.4\% showed a partial response and 28.6\% had progressive disease [10]. Similarly, in previously reported series, the combination of 5-fluorouracil, cisplatin and calcium folinate achieved a partial response rate of $67 \%$ in advanced LELC of the lung [20]. The addition of calcium folinate to 5-fluorouracil, as in the present chemotherapy regimen, has been shown to enhance the effect of 5-fluorouracil [20].

The present case demonstrates the rare association between silicosis and lymphoepithelioma-like carcinoma of the lung in which silica dust exposure might have been contributory to carcinogenesis. An interesting observation was the degree of amorphous and eccentric calcification within the mass, suggesting the presence of progressive massive fibrosis, at least initially. Subsequent development of lymphoepitheliomalike carcinoma would have resulted in uneven soft tissue growth, causing eccentricity of the calcification. Advanced lymphoepithelioma-like carcinoma of the lung has previously been described to comprise large relatively well-defined tumours closely associated with the mediastinum [11]. Despite the relatively high prevalence of Epstein-Barr virus infection among the Chinese, and the relatively high frequency of silicosis, there have not been any previous reports on an association between lymphoepithelioma-like carcinoma and silicosis. The potential carcinogenic effects of silica [5, 21-23] could have contributed to the development of lymphoepithelioma-like carcinoma in the present case, albeit probably not as a strong trigger. It is also possible that previous cases of nonsmall cell lung cancer in silicosis could have been lymphoepithelioma-like carcinoma misdiagnosed by unwary pathologists or clinicians. The good clinical response in the present case of lymphoepithelioma-like carcinoma of the lung to a combination of 5-fluorouracil, cisplatin, and calcium folinate warrants further studies.

\section{References}

1. Jindal SK, Aggarwal AN, Gupta D. Dust-induced interstitial lung disease in the tropics. Curr Opin Pulm Med 2001; 7: 272-277.

2. Finkelstein MM. Silica, silicosis, and lung cancer: a risk assessment. Am J Ind Med 2000; 38: 8-18.

3. Gift JS, Faust RA. Noncancer inhalation toxicology of crystalline silica: exposure-response assessment. J Expo Anal Environ Epidemiol 1997; 7: 345-358.

4. Markowitz G, Rosner D. The limits of thresholds: silica and the politics of science, 1935 to 1990. Am J Public Health 1995; 85: 253-262.

5. Cocco P. Multifactorial aetiology of lung cancer among silica-exposed workers. Ann Acad Med Singapore 2001; 30: 468-474.

6. Hessel PA, Gamble JF, Gee JB, et al. Silica, silicosis, and lung cancer: a response to a recent working group report. J Occup Environ Med 2000; 42: 704-720.

7. Begin LR, Eskandari J, Joncas J, Panasci L. Epstein-Barr virus related lymphoepithelioma-like carcinoma of lung. J Surg Oncol 1987; 36: 280-283.

8. Chan JKC, Hui PK, Tsang WYW, et al. Primary lymphoepithelioma-like carcinoma of the lung. Cancer 1995; 76: 413-422.

9. Wong MP, Chung LP, Yuen ST, et al. In situ detection of Epstein-Barr virus in non-small cell lung carcinomas. J Pathol 1995; 177: 233-240.

10. Chan TC, Teo ML, Lam KC, et al. Multimodality treatment of primary lymphoepithelioma-like carcinoma of the lung. Cancer 1998; 83: 925-929.

11. Ooi GC, Ho JCM, Khong PL, Wong MP, Lam WK, Tsang KWT. Computed tomography characteristics of advanced primary pulmonary lymphoepithelioma-like carcinoma (LELC). Eur Radiol 2003; 13: 522-526.

12. Butler AE, Colby TV, Weiss L, Lombard C. Lymphoepithelioma-like carcinoma of the lung. Am J Surg Pathol 1989; 13: 632-639.

13. Frank MW, Shields TW, Joob AW, et al. Lymphoepitheliomalike carcinoma of the lung. Ann Thorac Surg 1997; 64: 11621164.

14. Gal AA, Unger ER, Koss MN, Yen TS. Detection of Epstein-Barr virus in lymphoepithelioma-like carcinoma of the lung. Mod Pathol 1991; 4: 264-268.

15. Ferrara G, Nappi O. Lymphoepithelioma-like carcinoma of the lung. Two cases diagnosed in Caucasian patients. Tumori 1995; 81: 144-147.

16. Miller B, Montgomery C, Watne A, Johnson D, Bailey T, Kowalski R. Lymphoepithelioma-like carcinoma of the lung. J Surg Onco 1991; 48: 62-68.

17. Wockel W, Hofler G, Popper HH, Morresi A. Lymphoepithelioma-like carcinoma of the lung. Path Res Pract 1995; 191: 1170-1174.

18. Higashiyama M, Doi O, Kodama K, et al. Lymphoepitheliomalike carcinoma of the lung: analysis of two cases for EpsteinBarr virus infection. Hum Pathol 1995; 26: 1278-1282.

19. Curcio LD, Cohen JS, Grannis FW, Paz IB, Chilcote R, Weiss LM. Primary lymphoepithelioma-like carcinoma of the lung in a child. Chest 1997; 111: 250-251.

20. Ho JC, Lam WK, Ooi GC, Lam B, Tsang KW. Chemoradiotherapy for advanced lymphoepithelioma-like carcinoma of the lung. Respir Med 2000; 94: 943-947.

21. Ishihara $\mathrm{Y}$, Iijima $\mathrm{H}$, Matsunaga $\mathrm{K}$, Fukushima $\mathrm{T}$, Nishikawa T, Takenoshita S. Expression and mutation of p53 gene in the lung of mice intratracheal injected with crystalline silica. Cancer Lett 2002; 177: 125-128.

22. Liu B, Guan R, Zhou P, et al. A distinct mutational spectrum of $\mathrm{p} 53$ and K-ras genes in lung cancer of workers with silicosis. J Environ Pathol Toxicol Oncol 2000; 19: $1-7$.

23. Pairon JC, Brochard P, Jaurand MC, Bignon J. Silica and lung cancer: a controversial issue. Eur Respir J 1991; 4: 730744 .

24. Cocco P, Rice CH, Chen JQ, McCawley MA, McLaughlin JK, Dosemeci M. Lung cancer risk, silica exposure, and silicosis in Chinese mines and pottery factories: the modifying role of other workplace lung carcinogens. Am J Ind Med 2001; 40: 674-682.

25. Carta P, Aru G, Manca P. Mortality from lung cancer among silicotic patients in Sardinia: an update study with 10 more years of follow up. Occup Environ Med 2001; 58: 786793. 